\title{
BRUXISMO: UMA REVISÃO DA LITERATURA
}

\section{BRUXISM: A LITERATURE REVIEW}

\author{
Cathleen Kojo Rodrigues ${ }^{1}$, Rafael Gomes Ditterich ${ }^{2 *}$, \\ Ricardo Lima Shintcovsk ${ }^{3}$, Orlando Tanaka ${ }^{4}$ \\ 1 Faculdade de Odontologia de Piracicaba - UNICAMP, Programa de Pós-Graduação \\ em Odontologia em Saúde Coletiva, Piracicaba, SP \\ 2* Autor para contato: Universidade Federal do Paraná - UFPR, Curitiba, PR, Brasil; \\ (41) 9121-9190; e-mail: rafael.gomes@universia.com.br \\ 3 Cirurgião-dentista \\ 4 Pontifícia Universidade Católica do Paraná - PUCPR, Departamento de Odontologia, \\ Curitiba, PR
}

Recebido para publicação em 25/11/2005

Aceito para publicação em 21/06/2006

\section{RESUMO}

O bruxismo é definido como o contato estático ou dinâmico dos dentes, em momentos outros que não aqueles que ocorrem durante as funções normais da mastigação ou deglutição, e está sempre associado a um estado emocional alterado do paciente, ou seja, o stress. Este hábito parafuncional constitui um dos mais difíceis desafios para a odontologia restauradora e, em alguns casos, produz reflexos no periodonto, nos músculos mastigatórios, na articulação temporomandibular. Pode também causar dor de cabeça, efeitos comportamentais e psicológicos, sendo muito difícil sua resolução, dependendo da gravidade do desgaste produzido. Seus sinais e sintomas devem ser diagnosticados em fase precoce uma vez que a maioria dos danos provocados é irreversível. A etiologia não é bem entendida sendo uma desordem complexa e multifatorial e, com freqüência, difícil de ser identificada. Diferentes correntes tentam explicá-la, de tal forma que este artigo apresenta uma revisão da literatura sobre o bruxismo e descreve a sua etiologia, classificação, diagnóstico e condutas clínicas.

Palavras-chave: bruxismo, terapia miofuncional , oclusão dentária

\begin{abstract}
Bruxism is defined as the static or dynamic teeth contact, which does not occur during the normal functions of mastication or deglutition, and is always associated with a patient's altered emotional state, that is, stress. This parafunctional habit is one of the greatest challenges for Restoring Dentistry and, in some cases,
\end{abstract}


it affects the periodontium, the mastication muscles and the temporomandibular articulation. It can also cause headaches and comportamental and psychological effects, which turn out to be hard to treat, according to the gravity of the abrasion produced. Its signs and symptoms must be diagnosed in a precocious phase, since most of the damages are irreversible. However, its etiology is not well known, that is why it is considered a complex and multifactorial disorder, frequently hard to be identified. Different theories try to explain it, thus this paper presents a literature review on bruxism and describes its etiology, classification, diagnosis and clinical procedures.

Key words: bruxism, myofunctional therapy, dental occlusion

\section{Introdução}

Além dos movimentos funcionais, como a mastigação e a deglutição, pode-se encontrar também comportamentos parafuncionais representados algumas vezes pelo hábito de apertar e / ou ranger os dentes, conhecido como bruxismo (Attanasio, 1991; Dawson, 1993; Okeson, 1994; Zuanon et al., 1999).

O bruxismo é definido como o contato estático ou dinâmico da oclusão dos dentes em momentos outros, que não aqueles que ocorrem durante as funções normais da mastigação ou deglutição. É um fenômeno que ocorre preferencialmente durante o sono, de forma inconsciente, manifestando-se sob a forma de “apertamento" ou “ranger” de dentes (Arnold, 1981; Guzman e Kovalski, 1986; Medeiros, 1991; Bahlis et al., 1999; Teixeira et al., 1994; Uetanabara e Mazzetto, 2000). Este fenômeno não é um privilégio da civilização moderna, pois na Bíblia há menção ao ranger de dentes nos Salmos de David e no Evangelho de Matheus, cerca de 600-200 a.C. e 75-90 d.C., respectivamente (Teixeira et al., 1994), estando também associado ao sofrimento e punição divina (Attanasio, 1991). Está sempre associado a um estado emocional alterado do paciente, ou seja, o stress. Ocorre um elevado estado de alerta mental e físico, grande ansiedade, tensão e nervosismo (Leles e Melo, 1995; Uetanabara e Mazzetto, 2000).

O termo bruxismo vem do grego "bruchein”, que significa apertamento, friç̧ão ou atrito dos dentes sem finalidades funcionais. Foi utilizado pela primeira vez na literatura odontológica em 1907, como "Bruxomania”, e foi substituído em 1931 por “Bruxismo” (Mejias,
1982; Pavone, 1985; Attanasio, 1991). Ao longo do tempo já foi denominado: neurose do hábito oclusal, neuralgia traumática, ranger de dentes, efeito de Karolyi e mais recentemente parafunção (Ramfjord e Ash, 1984; Bahils et al., 1999). Atualmente, o verbete "bruxismo" é usado amplamente para descrever todos os tipos de ranger e apertamento de dentes (Shatkin, 1992; Teixeira et al., 1994; Attanasio, 1997; Bahlis et al., 1999).

A duração destes episódios é bastante variável, não somente de pessoa para pessoa como na mesma pessoa. O tempo de apertamento e ranger de dentes durante a noite podem variar de 5 a 38 minutos por noite e durante a atividade parafuncional a força de contato dentário chega a ser superior a três vezes àquela da atividade funcional do sistema mastigatório, podendo causar colapso das estruturas envolvidas (Okeson, 1992).

Silva et al. (1998) descrevem o bruxismo como uma atividade motora orofacial durante o sono, que é caracterizada por repetidas ou sustentadas contrações dos músculos elevadores da mandíbula, contrações que podem desenvolver rigor muscular vigoroso, em torno de 150 a $340 \mathrm{Kg}$ de carga puntiforme durante os períodos ativos, tendo, como conseqüência, fratura e desgaste dos dentes, problemas peridodontais, dor e fadiga muscular e dores de cabeça. E ainda, os autores afirmam que possivelmente todas as pessoas tenham breves períodos de bruxismo noturno em alguma época da vida, mas a maioria dos pacientes desconhece seu hábito.

O presente trabalho de revisão de literatura tem por objetivo verificar os aspectos clínicos do bruxismo, 
descrevendo a sua etiologia, classificação, diagnóstico e terapêutica.

\section{Etiologia}

A etiologia do bruxismo não é bem entendida, sendo uma desordem complexa e multifatorial e com freqüência, difícil de ser identificada. Existem diferentes correntes que tentam explicar a etiologia do bruxismo. A condição tem sido freqüentemente associada ao stress emocional e problemas oclusais ou a uma combinação de ambas (Pavone, 1985; Teixeira et al., 1994; Attanasio, 1997; Bailey, 1997; Watts, 1999; Bahlis et al., 1999; Uetanabara e Mazzetto, 2000). Por outro lado, a interferência oclusal não está sendo considerada mais o fator principal etiológico do bruxismo, enquanto que o estado emocional é considerado agora apenas parte da etiologia (Clark, 1985).

Segundo Pavone (1985) e Teixeira et al. (1994), os fatores etiológicos podem ser classificados em: dentais; psicoemocionais; sistêmicos; ocupacionais; idiopáticos; e nutricionais. Os fatores de origem dental como o tipo de oclusão, posição dental, mobilidade, padrão de movimento mandibular, morfologia, contatos prematuros e restaurações, teriam influência direta na etiologia do bruxismo (Arnold, 1981; Teixeira et al., 1994; Zuanon et al., 1999). O psicoemocional é apontado como um dos mais importantes na gênese do bruxismo, podendo estar relacionado com as frustrações de pacientes bruxomanos, que tendem a usar o seu aparelho estomatognático para descarregar seus sentimentos de agressividade (Ramfjord e Ash, 1987). Já os fatores ocupacionais, estão relacionados com a atividade física, profissional e mental, enquanto como condições sistêmicas relatam-se alterações no trato digestivo, como paralisias intestinais e desequilíbrio enzimáticos na digestão, que levam a estresse abdominal crônico, disfunções urológicas e desordens endócrinas, como o hipotiroidismo (Marks, 1980; Teixeira et al., 1994).

Já os fatores nutricionais como o consumo de bebidas xânticas (café, chá, chocolate, refrigerante tipo cola) e o tabaco estariam envolvidos, visto que eles estimulariam o sistema nervoso central, aumentando a ansiedade e o stress, conseqüentemente, desencadea- riam o bruxismo (Maciel, 1996), além de drogas como as anfetaminas, álcool também seria um dos fatores desencadeantes (Bailey, 1997; Uetanabara e Mazzetto, 2000).

A hereditariedade também é citada como fator que influencia na etiologia do bruxismo (Marks, 1980; Uetababara e Mazzetto, 2000). Em outro estudo, a persistência do bruxismo é de 23\% em gêmeos monozigotos (Uetanabara e Mazzetto, 2000).

\section{Classificação}

Segundo a classificação internacional de desordens do sono, o bruxismo pode ser classificado em moderado, de severidade moderada a severa, onde ocorrem evidências de danos às estruturas do sistema estomatognático (Bailey, 1997).

O bruxismo também é classificado como cêntrico e excêntrico. O bruxismo cêntrico está relacionado ao apertamento maxilo-mandibular. Ocorre geralmente durante o sono, num movimento isométrico, em relação de oclusão cêntrica ou máxima intercuspidação, sem que ocorra deslizamento. O bruxismo excêntrico caracteriza o rangimento, onde há apertamento e deslizamento dos dentes nas posições protusivas e lateroprotusivas. Há um padrão isotônico de contração muscular, apresentando, em função do deslizamento, desgaste tanto em dentes anteriores como em posteriores (Ortega, 1988; Bahlis et al., 1999).

Para Maciel (1996) e Bahlis et al. (1999), o bruxismo pode ser classificado ainda em crônico e agudo. O crônico é aquele no qual há uma adequação biológica e funcional do organismo e o agudo surge quando por alguma razão o processo se torna agressivo e ultrapassa a capacidade biológica de adaptação e defesa do sistema, gerando sensibilidade dentinária, pulpites e até uma necrose pulpar e perda da dimensão vertical.

Mohl (1989); Molina (1989); Okeson (1992), classificaram em bruxismo diurno e noturno, cada qual com etiologias distintas. A atividade parafuncional diurna consiste em apertar os dentes, ranger e em ter muitos hábitos bucais realizados sem que o indivíduo tenha consciência deles, como morder a língua e a bochecha, chupar dedos, morder objetos, como canetas e lápis. 
É comum o indivíduo manter os dentes em contato e aplicar grande força. Algumas atividades parafuncionais diurnas estão intimamente relacionadas com o trabalho que se faz e os indivíduos não sabem de seus hábitos de ranger ou morder bochechas. A atividade parafuncional durante o sono compõe-se de episódios únicos e contrações rítmicas. Se estas atividades resultam de diferentes fatores etiológicos ou do mesmo fenômeno manifestando-se de duas maneiras diferentes, não se sabe. Por isso o apertamento dos dentes e o bruxismo são, geralmente, referidos como bruxismo. Os eventos de bruxismo estão associados com a mudança do sono profundo para o sono leve, em geral, no estágio de sono mais leve 1 e 2 do sono não-REM (rapid eye moviment). Segundo Ingrassia Tonelli et al. (2005) a maior atividade muscular é encontrada no estado do sono e durante o despertar.

\section{Diagnóstico diferencial e conseqüências}

O diagnóstico e a avaliação clínica do bruxismo são complexos. Tanto bruxomanos quanto indivíduos normais podem apresentar alguma atividade parafuncional noturna (Rugh et al., 1984). O que diferencia é a duração e a intensidade das contrações musculares envolvidas, drasticamente alterada no paciente com bruxismo (Droukas et al., 1984; Kydd e Daly, 1985).

Os sinais e os sintomas do bruxismo devem ser diagnosticados precocemente uma vez que a maioria dos danos provocados são irreversíveis, por esta razão o profissional deve estar atento durante o exame clínico e conduzir uma anamnese detalhada (Bahlis et al., 1999).

Os principais danos ocasionados pelo bruxismo podem ser agrupados em: efeitos na dentição, no periodonto, nos músculos mastigatórios, na articulação temporo-mandibular (ATM), dor de cabeça, efeitos comportamentais e psicológicos (Glaros, 1977; Teixeira et al., 1994; Bahlis et al., 1999; Uetanabara e Mazzetto, 2000).

Durante a anamnese, o paciente freqüentemente relata fadiga e dor muscular causando a limitação da abertura da boca e ruídos na ATM. O paciente também informa ter sido alertado por pessoas de seu convívio, a respeito do ruído de ranger de dentes durante o seu sono, além de reclamar de aspereza das superfícies dentárias ehipersensibilidade térmica. Ao exame clínico, o sinal mais óbvio da presença do hábito parafuncional é o desgaste das bordas incisais e ou oclusais dos dentes antagônicos, visível geralmente na face incisal dos dentes anteriores e como facetas nas cúspides e restaurações posteriores. Também se observa a fratura dos dentes e restaurações (Maciel, 1996; Thompson et al., 1994; Teixeira et al., 1994; Attanasio, 1997).

Outros sinais e sintomas que indicam a presença do hábito parafuncional seriamhipermobilidade dentária na ausência de doença periodontal, pulpites, odontalgia (com polpa normal), fraturas parciais de coroas e migração de dentes. O dano provocado pode estar restrito a um único dente, a uma região ou toda a boca. Pode ocorrer também hipertonicidade dos músculos mastigatórios e dores de cabeça na região do músculo temporal (Molina, 1989; Teixeira et al., 1994; Maciel, 1996; Attanasio, 1997; Bahlis et al., 1999; Zuanon et al., 1999).

Como as alterações no periodonto decorrentes do bruxismo estão a reabsorção óssea (lesão traumática), alargamento do espaço periodontal, necrose dos tecidos periodontais, mobilidade, formação de diastemas, recessão gengival e hipercementose (Ramfjord e Ash, 1987; Teixeira et al., 1994; Cabre, 1995).

Radiograficamente, pode-se observar o desaparecimento da lâmina dura, alteração do espaço periodontal, que tanto pode desaparecer quanto apresentar-se aumentado, reabsorção radicular, fraturas radiculares e cálculos pulpares (Arnold, 1981; Thompson et al., 1994; Teixeira et al., 1994; Zuanon et al., 1999).

Sobre a musculatura o bruxismo pode provocar o aumento no tônus e na atividade muscular, hipertonismo dos músculos mastigatórios, sobretudo o masseter, contraturas e espasmos musculares, podendo desencadear um quadro de miosite (Burstone, 1946; Thompson et al., 1994; Maciel, 1996; Bahlis et al., 1999). A sintomatologia miofascial é caracterizada por dor muscular regional e presença de pontos sensíveis localizados, que se evidenciam quando palpados manualmente (Leles e Melo, 1995).

Em casos severos, o músculo hipertrofiado pode causar contração facial, trismo e alterações oclusais, alterando o padrão de abertura e de fechamento da 
mandíbula, ocasionando problemas na fala e mastigação (Muller, 1982).

Os reflexos do bruxismo na articulação temporomandibular estão diretamente relacionados às mudanças que ocorrem na musculatura. A dor e o desconforto nessa articulação são as principais queixas do paciente, além de dificuldades mastigatórias, travamento articular, falta de coordenação durante os movimentos mandibulares, luxação, subluxação, crepitação, ruído articular uni ou bilateral, alterações degenerativas na articulação, limitação de abertura, restrição dos movimentos e desvios na trajetória de abertura (Glaros e Rao, 1977; Ramfjord, 1984; Zuanon, 1999; Teixeira et al., 1994; Bahlis et al., 1999).

A dor de cabeça sentida como uma pressão discreta na região da fronte, atrás dos olhos e ao longo da origem do masseter no arco zigomático é o sintoma mais comum. Também aparece como dor unilateral como na enxaqueca, porém sem os distúrbios neurológicos advindos dela (Berlin, 1961; Thompson et al., 1994; Teixeira et al., 1994; Maciel, 1996; Attanasio, 1997). Mais freqüentemente ocorre a cefaléia de contração muscular, caracterizada por uma dor bilateral que dura várias horas (Maciel, 1996).

A dor de cabeça surge como conseqüência da isquemia produzida pelo estado hipertônico dos músculos, pois a crescente falta de oxigenação estimula as terminações nervosas que respondem com a dor (Berlim, 1961; Teixeira et al., 1994).

\section{Terapêutica}

O tratamento deve ser realizado de forma multidisciplinar, com a participação da Odontologia, Psicologia, Fisioterapia e Fonoaudiologia, abrangendo aspectos dentários, médicos e psicológicos do paciente (Mazzetto et al., 1995 apud Zuanon et al., 1999).

Por ser o bruxismo uma parafunção multifatorial não há uma forma de tratamento capaz de eliminá-lo permanentemente (Ingrassia Tonelli et al., 2005). O profissional deve analisar a etiologia da disfunção para determinar o tratamento que elimine os fatores causais. O plano de tratamento deve atender aos seguintes objetivos: redução da tensão física e psicológica, tratamento dos sinais e sintomas, minimização de interferências oclusais e rompimento do padrão neuromuscular habitual (Mikami, 1977; Travell e Simons, 1983; Pavone, 1985; Okeson, 1992; Teixeira et al., 1994; Leles e Melo, 1995).

Segundo Graf (1969) o ponto de partida do tratamento é a tentativa de diminuição do stress psicológico do paciente, por meio de exercícios de relaxamento, massagem e fisioterapia. Esse tratamento diminui os sintomas e não a causa, pois, o hábito pode retornar sempre que o paciente tiver diminuído a sua tolerância para com a desarmonia oclusal (Ramfjord e Ash, 1984).

O tratamento específico para a dor muscular é acompanhado de modalidades que interrompem o mecanismo do ciclo da dor, como terapia do ponto de desencadeamento miofascial (spray de vapor frio), bloqueio anestésico, associado a modalidades fisioterápicas: exercícios para restaurar a função (isotônicos, isométricos e de coordenação), massagem e calor profundo (Bell, 1990; AAOP, 1993; Leles e Melo, 1995).

A terapia oclusal pode envolver o ajuste oclusal. Embora, a condição oclusal exerça pequena influência sobre o processo, o ajuste oclusal, método terapêutico irreversível, é indicado para minimizar danos gerados pelo apertamento ou ranger de dentes e não para o tratamento do bruxismo. Deve ser usado somente quando o desgaste causado pelo bruxismo não tenha alterado a dimensão vertical. O tratamento restaurador é realizado quando há uma oclusão harmoniosa não podendo ser alcançada apenas por ajuste oclusal, devido à severidade do desgaste que chegou a causar perda de estrutura dental (Teixeira et al., 1994).

O controle do comprometimento muscular e oclusal pode ser atingido com o uso de placas interoclusais, também denominadas placas oclusais ou placa mio-relaxante total (Ramjford, 1987; Attanasio, 1991; Dawson, 1993; Okeson, 1994; Leles e Melo, 1995; AAOP, 1996). Esta placa deve ser feita com um material rígido, com superfície oclusal plana, ajustado em relação cêntrica, com características de uma oclusão mecanicamente normal. Ela recobre todos os dentes, alterando o relacionamento oclusal e redistribui forças, prevenindo desgastes e mobilidade dental, reduzindo a parafunção noturna e alterando o padrão neuromuscular e o relacionamento articular (Travel e Simons, 1983; AAOP, 1993; Holmgren e 
Sheikholeslam, 1994; Leles e Melo, 1995).

O uso de medicamentos (relaxantes musculares) para diminuir a tensão do paciente e conseqüentemente o bruxismo é um tratamento de eficácia temporária. Uma vez suspensa a medicação, o hábito parafuncional se reinstalará, além de apresentar o risco de dependência química (Rugh e Solberg, 1975; Montgomery, 1986; Teixeira et al., 1994; Zuanon et al., 1999).

Vários estudos sugerem a eficácia do propanolol, benzodiazepínicos e bromocriptine no tratamento do bruxismo (Sjoholm et al., 1996; Attanasio, 1997; Lobbezoo et al., 1997; Uetanabara e Mazzetto, 2000).

Recente descoberta em termos de tratamento químico é a Toxina Botulínica (BTX), uma potente toxina biológica, segura e efetiva no tratamento de diversas formas de desordens neurológicas. Ela é produzida por uma bactéria anaeróbia chamada Clostridium botulinum com efeitos paralisantes devido a sua capacidade de inibir a liberação de acetilcolina nas junções neuromusculares. Seus efeitos duram cerca de 3 a 6 meses, devendo ser injetado, somente, 400 UM de BTX A, a cada 3 meses. Este tratamento apresenta desvantagens como o alto custo, requer profissional que domine conceitos de farmacologia e com bom conhecimento de anatomia, e deve ser indicado para casos de bruxismo severo (Tan, 2000).

\section{Discussão}

A determinação da prevalência do bruxismo na população geral é difícil, pois este hábito é realizado inconscientemente por muitos indivíduos (Glaros, 1981; Pavone, 1985; Ramjford, 1987; Arita et al., 1990; Attanasio, 1991; Mohl, 1989; Okeson, 1992). A prevalência na população varia de 15 a $90 \%$ em adultos e de 7 a 88\% em crianças (Attanasio, 1991; Zuanon et al., 1999), e há a influência étnica para o desenvolvimento do bruxismo, em que a população afroamericana apresenta uma porcentagem de 9,4\%, a asiática de $24,6 \%$, a euro-americana de $23,1 \%$ e a hispânica de 16,9\% (Uetanabara e Mazzetto , 2000).

Para Leles e Melo (1995), na grande maioria ocorrem episódios transitórios, não desenvolvendo alterações significativas. No entanto, cerca de 10 a 15\% apresentam problemas clínicos que merecem atenção profissional, como desgastes dentais acentuados, mialgias e desordens articulares, com envolvimento periodontal.

A duração destes episódios é bastante variável, não somente de pessoa para pessoa como na mesma pessoa. O tempo de apertamento e ranger de dentes durante a noite podem variar de 5 a 38 minutos por noite e durante a atividade parafuncional a força de contato dentário chega a ser superior a três vezes àquela da atividade funcional do sistema mastigatório, podendo causar colapso das estruturas envolvidas (Trenouth, 1979; Reding et al., 1968 apud Okeson, 1992).

A condição tem sido freqüentemente associada ao stress emocional e a problemas oclusais ou a uma combinação de ambas (Pavone, 1985; Teixeira et al., 1994; Attanasio, 1997; Bailey, 1997; Watts, 1999; Bahlis et al., 1999; Uetanabara e Mazzetto, 2000), e a interferência oclusal não está sendo considerada mais o fator principal etiológico do bruxismo, e o estado emocional é considerado agora apenas parte da etiologia (Clark, 1985).

Entretanto, pesquisas atuais têm mostrado que fatores psicológicos não são os principais fatores, mas apenas os agravantes ou perpetuadores. A Universidade de Montreal, no Canadá num estudo de variabilidade em pacientes com Bruxismo para identificar a correlação entre o distúrbio e a ansiedade, foi verificado que em $48 \%$ dos casos estudados a ansiedade tinha relação com o distúrbio (Silva, 2003).

É ponto pacífico que a etiologia do bruxismo é multifatorial, portanto, complexa, e que não há como eliminá-lo permanentemente. Este hábito parafuncional constitui um dos mais difíceis desafios para a odontologia restauradora, sendo muito difícil sua resolução dependendo da gravidade do desgaste produzido (Pavone, 1985; Mohl et al., 1989; Dawson, 1993).

Devido a tais características têm-se optado por tratamentos não invasivos e reversíveis capazes de aliviar seus sintomas. Geralmente, é suficiente o controle das causas primárias (stress e ansiedade), dos efeitos principais (mialgia e atrição dentária) e fatores conjugados como o apertamento dentária (Kaplan e Assael, 1991; Leles e Melo, 1995; Uetanabara e Mazzetto, 2000).

Seguindo essa filosofia, as placas inter-oclusais 
figuram como a primeira opção no tratamento do bruxismo, pois, podem atuar na articulação temporomandibular induzindo o côndilo a se posicionar corretamente na fossa condilar (Zuanon et al., 1999). Entretanto, não há evidências de que eliminem o bruxismo definitivamente, ocorrendo muitas vezes recidiva (Okeson, 1992; Okeson et al., 1994; Travell et al., 1883; Clark, 1984; Attanasio, 1991; Leles e Melo,1995)

O efeito das placas interoclusais em pacientes com bruxismo, revelou que as placas não eliminavam o hábito parafuncional (Holmgren et al., 1993). Na maioria das vezes, ocorre recidiva do bruxismo após a suspensão de um tratamento a longo prazo com placas interoclusais (Travel e Simons, 1983; Okeson, 1992; Holmgren et al., 1993; Holmgren e Sheikholeslam, 1994).

Embora a utilização da placa diminua de forma imediata a atividade eletromiográfica e sintomatologia aguda não há evidências que a diminuição da atividade muscular se mantenha a longo prazo (Clark, 1984; Leles e Melo, 1995).

A terapia farmocológica não é amplamente utilizada por ter efeito somente durante o uso da droga e por apresentar riscos de dependência química (Muller et al., 1982; Montgomery, 1986; Teixeira et al., 1994; Zuanon et al., 1999).

Em outra linha de pesquisa, a avaliação de vários neurotransmissores cerebrais nos pacientes com bruxismo mostra um ligeiro desequilíbrio. Isso é o que sugere a teoria neurológica - os neurotransmissores são responsáveis pela transmissão de mensagens de um neurônio para outro. Pacientes com bruxismo têm uma produção maior de dopamina (Silva, 2003).

Os ajustes oclusais devem ser bem ponderados já que seus efeitos são irreversíveis (Aaop, 1993; Griffiths, 1983; Clark e Adler, 1985; Leles e Melo, 1995), embora seja o método preferido de muitas escolas (Ramfjord, 1961; Zuanon et al., 1999; Bahlis et al., 1999).

Recomenda-se a conscientização do paciente pelo profissional quanto à inter-relação entre o hábito e estados emocionais alterados (Rugh e Solberg, 1976; Moss et al., 1982; Teixeira et al., 1994; Uetanabara e Mazzetto, 2000). A retroalimentação biológica ou "biofeedback” que consiste na conscientização do paciente para que ele próprio seja capaz de identificar e controlar a hiperatividade muscular derivada do bruxismo é capaz de reduzir a freqüência e duração da desordem funcional (Moss et al., 1982), mas não deve ser utilizada como tratamento isolado (Teixeira et al., 1994).

A associação entre o uso de placas oclusais e exercícios de relaxamento, massagem e fisioterapia alcançam bons resultados na redução da sintomatologia dolorosa.

Por fim, observou-se que não há uma padronização dos experimentos realizados que relatam sucesso no tratamento do bruxismo que tornem confiáveis a adoção de novas condutas.

\section{Conclusão}

O bruxismo apresenta características próprias que variam individualmente, portanto, a terapêutica deve ser conservadora, reversível e não invasiva. Devese realizar um gerenciamento da condição do paciente, que destaque a atuação multidisciplinar (Odontologia, Psicologia, Fisioterapia e Fonoaudiologia).

\section{REFERÊNCIAS}

1. AMERICAN ACADEMY OF OROFACIAL PAIN. Temporomandibular Disorders: Guidelines for classification, assessment and management. 2. ed. Chicago: Quintessence, 1993.

2. AMERICANACADEMY OF OROFACIAL PAIN. Orofacial Pain-Guidelines for assessment, diagnosis, and management. Quintessence, 1996.

3. ARITA, C. A.; CARVALHO, P. C. L.; SILVA, M. A. M. R.; BATAGLION, C.; CHAGURI, N. A.; NUNES, L. J. Alterações provocadas pelo bruxismo sobre o sistema estomatognático A importância de seu diagnóstico. Rev Gaúcha de Odontologia, v. 38, n. 4, p. 254-61, 1990.

4. ARNOLD, M. Bruxism and the occlusion. Dent Clin North Am, v. 25, p. 395-407, 1981.

5. ATTANASIO, R. Nocturnal bruxism and its clinical managment. Dent Clin North Am, v. 35, n. 1, p. 245-52, 1991.

6. ATTANASIO, R. An overview of bruxism and its management. Dent Clin North Am, v. 41, n. 2, p. 229-41, 1997.

7. BAHILS, A.; RODRIGUES, N. L.; FERRARI, E. Bruxismo. Revista Odonto Ciência, n. 27, p. 7-20, 1999. 
8. BAILEY, D. R. Sleep disorders. Overview and relationship to orofacial pain. Dent Clin North Am, v. 41, n. 2, p. 189-209, 1997.

9. BELL, W. Temporomandibular Disorder: classification, diagnosis and management. 3. ed. Chicago: Year Book Med Publ, 1990.

10. BELL, W. Dores Orofaciais. 3. ed. Rio de Janeiro: Quintessence, 1990.

11. BERLIN, R. Bruxism and chronic headache. Dent Dig, v. 67, p. 32-44, 1961.

12. BURSTONE, M. J. Psychosomatics aspects of dental problems. J Am Dent Assoc, v. 62, p. 21-44, 1946.

13. CABRE, J. S. Bruxism y su repercusion clinica sobre la oclusion. Rev Actual Estomatol Esp, v.45, n. 343, p. 37-44, 1985.

14. CLARK, G. A critical evaluation of orthopedic interocclusal appliance therapy: effectiveness for specific symptoms. J Am Dent Assoc, v. 108, p. 363-68, 1984.

15. CLARK, G.; ADLER, R. A critical evaluation of occlusal therapy: occlusal adjustment procedures. J Am Dent Assoc, v. 110, n. 5, p. 743-50, 1985.

16. DAWSON, P. E. Avaliação, diagnóstico e tratamento dos problemas oclusais. 2.ed. São Paulo: Artes Médicas, 1993.

17. DROUKAS, B.; LINDEE, C.; CARLSSON, G. Relationship between occlusal factors and sings symptoms of mandibular dysfuncion. A clinical study of 48 dental students. Acta Odontol Scand, v. 42, p. 277-83, 1984.

18. GLAROS, A. G.; RAO, S. M. Effects of bruxism: Areview of the literature. J Prosthet Dent, v.38, p. 149-57, 1977.

19. GLAROS, A. G. Incidence of diurnal and nocturnal bruxism. J Prosthet Dent, v. 45, n. 5, p. 545-9, 1981.

20. GRAF, H. Bruxism. Dent Clin NAmer, v. 13, n. 3, p. 659-65, 1969.

21. GRIFFITHS, R. Report the President's Conference on the Examination. Diagnosis and Management of Temporomandibular Disorders. J Am Dent Assoc, v. 106, p. 757,1983 .

22. GUZMAN, E. E., KOVALSKI, G. S. Neurofisiologia de la oclusion. Monserrate, 1986.

23. HOLMGREN, K., SHEIKHOLESLAM, A.; RIISE, C. Effect of a full-arch maxillary occlusal splint on parafunctional activity during sleep in patients with nocturnal bruxism and signs and symptoms of craniomandibular disorders. J Prosthet Dent. v. 69, p. 293-7, 1993.

24. HOLMGREN, K., SHEIKHOLESLAM, A. Occlusal adjustment and myoelectric activity of the jaw elevator muscles in patients with nocturnal bruxism and craniomandibular disorders. Scand J Dent Res, v. 102, p. 238-43, 1994.

25. INGRASSIA TONELLI, M. E.; RIVAROLA, E. M.;
BONGIOVANNI, I. Bruxismo nocturno. Rev Assoc Odontol Argent, v. 93, n. 1, p. 21-3, 2005.

26. KAPLAN,A.;ASSAEL, L. Temporomandibular disorders: diagnosis and treatment. Philadelphia: WB Saunders, 1991.

27. KYDD, D.; DALY. C. Duration of nocturnal tooth contacts during bruxism. J Prosthet Dent, v. 53, p. 717-21, 1985.

28. LELES, C. R.; MELO, M. Bruxismo e Apertamento DentalUma conduta clínica racional. ROBRAC- Rev Odontol Bras Central, v. 5, n. 15, p. 22-5, 1995.

29. LOBBEZOO, F.; SOUCY, J. P.; HARTMAN, N. G.; MONTPLAISIR, J. Y.; LAVIGNE, G. J. Effects of the D2 receptor agonist bromocriptine on sleep bruxism: report of two singlepatient clinical trials. J Dent Res, v. 76, n. 9, p. 1610-14, 1997.

30. MACIEL, R. N. Oclusão e ATM - Procedimentos clínicos. São Paulo: Santos, 1996.

31. MARKS, M. B. Bruxism in allergic children. Am J Orthod, v. 11, p. 48-59, 1980.

32. MEDEIROS, J. S. Oclusão. São Paulo: American Med, 1991.

33. MEJIAS, J. E.; MEHTA, N. R. Subjective and objective evaluation of bruxing patients un-dergoing shortterm splint therapy. J Oral Rehabil, v. 9, p. 279-89, 1982.

34. MIKAMI, D. B. A rewiew of psychogenic aspects and treatment of bruxism. J Prosthet Dent, v. 37, n. 4, p. 411-9, 1977.

35. MOHL, N. D.; ZARB, G. A.; CARLSSON, G. E. Fundamentos de oclusão. São Paulo: Quintessence, 1989.

36. MOLINA, O. F. Fisiopatologia craniomandibular - oclusão e ATM. São Paulo: Pancast, 1989.

37. MONTGOMERY, M. T. Etalii Effects of diazepam on nocturnal mastgatory muscle activity. J Dent Res, v. 65, p. 452-64, 1986.

38. MOSS, R. A. HAMMER, D.; ADAMS, H. E.; JENKINS, J. O.; THOMPSON, K.; HABER, J. A more efficient biofeedback procedure for the treatment of nocturnal bruxism. J Oral Rehabil, v.9, p. 125-31, 1982.

39. MULLER, E. et al. Bruxismo: revisión bibliografica. Acta Odont Venez, v. 20, n. 2, p. 115-42, 1982.

40. NADLER, S. C. The importance of bruxism. J Oral Med, v.23, p. 142-8, 1968.

41. OKENSON, J. P. Fundamentos de Oclusão e Desordens Temporo-mandibulares. 2. ed. São Paulo: Artes Médicas, 1992.

42. OKESON, J. P.; PHILLIPS B. A.; BERRY D. T.; BALDWIN, R. M. Nocturnal bruxing events: a report of normative data and cardiovascular response. J Oral Rehabil, v. 21, p. 623-30, 1994.

43. ORTEGA, A. C. Tratamiento del bruxismo. Rev Actual Estomatol Esp, v. 48, n. 374, p. 33-87, 1988.

Publ. UEPG Ci. Biol. Saúde, Ponta Grossa, 12 (3): 13-21, set. 2006 
44. PAVONE, B. W. Bruxism and its effects on the natural teeth. J Prosthet Dent, v. 53, n. 5, p. 692-6, 1985.

45. RAMFJORD, S. P. Bruxism: a clinical and EMG study. JAm Dent Assoc, v. 62, p. 21-44, 1961.

46. RAMFJORD, S. P.; ASH, M. M. Oclusion. Philadelphia: WB Saunders, 1966.

47. RAMFJORD, S. P.; ASH, M. M. Oclusão. 3. ed., São Paulo: Interamericana, 1984.

48. RAMFJORD, S. P.; ASH, M. M. Oclusão. 3. ed., Rio de Janeiro: Guanabara, 1987.

49. RUGH, J.; SOLBERG, W. K. Eletromyographical studies of bruxist behavior before and during treatment. Calif Dent Ass, v. 3, p. 57, 1975.

50. RUGH, J.; SOLBERG, W. K. Psychological implications in temporomandibular pain and dysfunction. Oral Sciences Rev, v. 7, p. 3-30, 1976.

51. RUGH, J.; BARGH, N.; DRAGO, C. Experimental occlusal discrepancies and nocturnal bruxism. J Prosthet Dent, v. 51, p. 548-53, 1984.

52. SILVA, L. G., BELESTRERI C, CUNHAL. T. B. V. Prevalência de bruxismo em alunos da odontologia. Rev Méd Hosp São Vicente de Paula, n. 10, v. 23, p. 5-8, 1998.

53. SILVA, S.R. Bruxismo. Rev Assoc Paul Cir Dent, v. 57, n. 6 , p. 409-17, 2003.
54. SJOHOLM, T. T.; LEHTINEN, I.; PIHA, S. J. The effect of propanolol on sleep bruxism: hypothetical considerations based on a case study. Clin Auton Res, v. 6, n. 1, p. 37-40, 1996.

55. TAN, E. K.; JANKOVIC, J. Treating Severe Bruxism with Botulinum Toxin. J Am Dent Assoc, v. 131, p.211-6, 2000.

56. TEIXEIRA, M.; RIBEIRO, C. P.; QUEIROZ, A.; PERDOMO, G. W. Bruxismo: O desgaste dental em resposta à interferência oclusal. ROBRAC- Rev Odontol Bras Central, v. 4, n. 13, p. 813, 1994.

57. THOMPSON, B. A.; BLOUNT, B. W.; KRUMHOLZ, T. S. Treatment approaches to bruxism. Am Fam Phys, v. 49, n. 7, p. 1617-22, 1994.

58. TRAVELL, J. G.; SIMONS, D. G. Myofascial Pain and Dysfuncion. The Trigger Point Manual. The Upper Extremities, Williams \& Wilkins, 1983.

59. UETANABARA, R.; MAZZETTO, M. O. Bruxismo: Uma visão atual. Rev Odontol UNICID, v. 12, n. 2, p. 163-169, 2000.

60. WATTS, M. W.; TAN, E. K.; JANKOVIC, J. Bruxism and cranial-cervical dystonia: is there a relationship? Cranio, v. 17, n. 3, p. 196-201, 1999.

61. ZUANON, A. C. C.; CAMPOS, C. G. A.; GIRO, E. M. A; PANSANI, C. A. Bruxismo Infantil. Odont Clin, v. 9, n. 1, p. 414, 1999. 